\title{
An Infection Preventionist's View of the Compendium of Strategies to Prevent Healthcare-Associated Infections: Structure, Process, and Outcome
}

\author{
Janet P. Haas, PhD, RN, CIC
}

The set of articles included in the 2008 "A Compendium of Strategies to Prevent Healthcare-Associated Infections in Acute Care Hospitals"1 was groundbreaking because the articles distilled the latest information about 6 of the most pressing healthcare-associated infection (HAI) prevention problems into a compelling framework, with guidance for immediate interventions. Further, they were all included as a supplement to the regular monthly issue of Infection Control and Hospital Epidemiology that could be kept neatly in healthcare professionals' offices for reference. Importantly, colleagues from all disciplines could access these articles online free of charge, along with patient educational material available in English, Spanish, and several additional languages. One central topic missing from the 2008 Compendium was hand hygiene, a cornerstone of infection prevention. The World Health Organization (WHO) Clean Care Is Safer Care initiative $^{2}$ was then the focus for hand hygiene monitoring and improvement, and it was unclear at that point whether another guidance document on the topic would have provided additional useful information.

The 2014 Compendium updates will continue to be useful to front-line infection preventionists (IPs), healthcare epidemiologists, and others involved in the prevention of HAIs. This time around, the 6 original Compendium topics (strategies for preventing methicillin-resistant Staphylococcus aureus [MRSA], Clostridium difficile infection [CDI], surgical site infection [SSI], ventilator-associated pneumonia [VAP], catheter-associated urinary tract infection [CAUTI], and central line-associated bloodstream infection [CLABSI]) have been joined by a new hand hygiene guidance document. ${ }^{3}$ The Society for Healthcare Epidemiology of America (SHEA), the Infectious Diseases Society of America (IDSA), and their partners recognized that, given the substantial number of publications since 2008 on this topic, incorporating up-todate evidence into a hand hygiene guidance document could be useful for healthcare providers. It was particularly re- warding for me to participate as a Compendium hand hygiene section co-lead and representative of the Association for Professionals in Infection Control and Epidemiology (APIC). APIC is one of the major Compendium partnering organizations, and the participation and leadership of APIC representatives in the Compendium advisory group and writing groups enabled infection preventionists to provide our perspectives and input throughout the Compendium writing process.

\section{STRUCTURE}

Writing a compendium is a huge undertaking and could be described as a massive group project. The comprehensive goals of the Compendium updates included review of relevant literature published since the 2008 articles, updating and categorizing recommendations as basic practices or special approaches, expanding on implementation strategies, and updating performance measures. A new conceptual framework for discussion of implementation strategies focused on engagement, education, execution, and evaluation as core components. The authors of each Compendium article have provided practical examples intended to promote and operationalize strategies and actions related to key recommendations.

SHEA and IDSA have described the basic steps used for the Compendium articles, ${ }^{4}$ but from the perspective of a colead of the hand hygiene article, here is some additional detail. This process was thoughtful from its inception. The goal was to use a similar organizational structure for each article to make the set of articles more reader friendly. For hand hygiene, the challenge was to fit a topic without a diagnostic component or easily correlated HAI outcome measures into this paradigm. We chose to focus our discussion on measurement of hand hygiene adherence and to use the best estimates of the impact of changes in hand hygiene adherence

Affiliation: 1. Westchester Medical Center Infection Prevention and Control and New York Medical College Department of Medicine, Valhalla, New York.

Received April 14, 2014; accepted April 16, 2014; electronically published July 16, 2014.

Infect Control Hosp Epidemiol 2014;35(8):961-963

(C) 2014 by The Society for Healthcare Epidemiology of America. All rights reserved. 0899-823X/2014/3508-0003\$15.00. DOI: 10.1086/677146 
on infection outcomes. There was a deliberate effort to include members of the partnering organizations as well as representatives from other professional societies with content expertise as part of the writing teams. The goal was to encourage ongoing dialogue among representatives of many stakeholder organizations throughout the writing, review, and editing process.

\section{PROCESS}

A key component of the process was regular discussions between lead authors and the SHEA and IDSA Compendium leads. This helped to keep the writing moving along and allowed us to learn from each other. The writing groups each included individuals from the front lines of infection prevention-IPs and healthcare epidemiologists-as well as representatives with expertise in quality improvement or the care of special patient populations (eg, pediatrics, critical care). All writing group members, including representatives from APIC and the other partnering organizations, were an integral part of the Compendium development.

A conscious effort was made to keep these articles as concise as possible while highlighting new information. The literature review was largely limited to publications since 2008 or, in the case of hand hygiene, since the 2002 Centers for Disease Control and Prevention (CDC) guideline. ${ }^{5}$ The writers had a huge body of knowledge that had to be distilled to just enough detail to be useful. To strike the right balance, drafts were reviewed by internal writing group members, the Compendium expert panel and advisory group, external subject matter experts, the APIC Practice Guidance Committee and the APIC Board of Directors, as well as the boards of the other partnering organizations. Comments from all groups were used to guide revisions. This iterative process contributed to the quality of the documents. In the case of hand hygiene, we appreciated the reminders to "keep it useful"- to give specific recommendations to improve care, to distill complex information into tables, and to limit the times when we said more research is needed.

Nevertheless, more research is still clearly needed for many aspects of infection prevention. Although the focus of the Compendium articles is utilitarian rather than academic, each article contains a section for unresolved issues. Future research on these issues from our infection prevention communities, including the APIC Research Committee and the SHEA Research Network, can help to better inform future prevention strategies. Attention also must be paid to study design and quality in order to improve our ability to optimize and prioritize HAI prevention efforts.

The modified GRADE system ${ }^{6.7}$ used to evaluate the quality of supporting evidence allowed us to recommend some interventions that lack evidence based on randomized control trials but where there was a body of observational studies with consistent results or strong expert consensus supporting benefits of the recommended practice along with a low likelihood of risks or unintended consequences. This flexibility is key for infection prevention since it is often unethical or highly impractical to conduct randomized control trials of basic interventions such as hand hygiene.

\section{OUTCOME}

What outcomes are expected from "A Compendium of Strategies to Prevent Healthcare-Associated Infections in Acute Care Hospitals: 2014 Updates"? The overarching goal is to decrease the risk of HAIs. For healthcare professionals in smaller settings and those with limited resources, these guidance documents can be lifelines to best practices. In settings with a focus on evidence-based practice (or those that need to develop such a focus), the literature review summaries and references provided by the Compendium writing groups are there to share with colleagues. The fact that SHEA and IDSA have recruited individuals with substantial expertise from diverse backgrounds to participate in the Compendium writing process will help to facilitate broad acceptance. Perhaps a follow-up survey would be helpful to assess the utility of these articles and to define opportunities for future improvements and expansion of topics.

APIC and its more than 15,000 members who serve as IPs in hospitals and other practice settings are committed to continuing to lead in implementing evidence-based best practices to protect our patients from harm. "A Compendium of Strategies to Prevent Healthcare-Associated Infections in Acute Care Hospitals: 2014 Updates" is a valuable resource, and APIC is proud to have collaborated with SHEA, IDSA, the American Hospital Association, and The Joint Commission to bring it to the infection prevention and control community. APIC thanks SHEA and IDSA for their leadership on this important initiative.

\section{ACKNOWLEDGMENTS}

Financial support. J.P.H. has received research funding from $3 \mathrm{M}$.

Address correspondence to Janet P. Haas, PhD, RN, CIC, Westchester Medical Center Infection Prevention and Control, Macy Pavilion SW-246, 100 Woods Road, Valhalla, NY 10595 (haasj@wcmc.com).

\section{REFERENCES}

1. Yokoe DS, Mermel LA, Anderson DJ, et al. A Compendium of strategies to prevent healthcare-associated infections in acute care hospitals. Infect Control Hosp Epidemiol 2008;29(suppl 1):S12-S21.

2. Pittet $D$, Allegranzi B, Boyce J. The World Health Organization guidelines on hand hygiene in health care and their consensus recommendations. Infect Control Hosp Epidemiol 2009;30(7):611-622.

3. Ellingson K, Haas JP, Aiello A, et al. Strategies to prevent healthcare-associated infections through hand hygiene. Infect Control Hosp Epidemiol 2014;35(8):937-960 (in this issue).

4. Yokoe DS, Anderson DJ, Berenholtz SM, et al. Introduction to "A Compendium of Strategies to Prevent Healthcare-Associated Infections in Acute Care Hospitals: 2014 Updates." Infect Control Hosp Epidemiol 2014;35(5):455-459.

5. Boyce JM, Pittet D. Guideline for hand hygiene in health-care 
settings: recommendation of the Healthcare Infection Control Practices Advisory Committee and the HICPAC/SHEA/APIC/ IDSA Hand Hygiene Task Force. Infect Control Hosp Epidemiol 2002;23(suppl 12):S3-40.

6. Guyatt $\mathrm{GH}$, Oxman AD, Vist GE, et al. GRADE: an emerging consensus on rating quality of evidence and strength of recommendations. BMJ 2008;336(7650):924-926.

7. GRADE. Canadian Task Force on Preventive Health Care website. http://canadiantaskforce.ca/methods/grade/. Accessed December 31, 2013. 